\title{
GENERALIZED NEAR-FIELDS
}

\author{
by C. V. L. N. MURTY
}

(Received 6th Ocwher, 1982)

\section{Introduction}

By analogy with the concept of "inverse semi-group" in semi-group theory, in this paper we introduce the concept of "generalized near-field" in near-rings. A near-ring $N$ is called a generalized near-field (GNF) if for each $a \in N$ there exists a unique $b \in N$ such that $a=a b a$ and $b=b a b$, that is $(N, \cdot)$ is an inverse semi-group. Surprisingly, this concept in rings coincides with that of "strong regularity". But this is not true in the case of near-rings. Every GNF is strongly regular, but in general the converse is not true.

The aim of this paper is to show that for any near-ring $N$ the following are equivalent.

(i) $N$ is a GNF.

(ii) $N$ is regular and each idempotent is central.

(iii) $N$ is fegular and subcommutative.

Also we prove that if $N$ is a near-ring with dcc on ideals, then $N$ is a GNF if and only if it is the direct sum of finitely many near-fields. (ii) is equivalent to $(N, \cdot)$ is a Clifford semi-group. See [2] for properties of inverse semi-groups.

Throughout this paper, $N$ stands for a right near-ring. For the basic terminology and notation we refer to [9]. Recall that a near-ring $N$ is called regular if for each $a \in N$, $a=a b a$ for some $b \in N$.

Lemma 1. If $N$ is a GNF, then $N$ is zerosymmetric.

Proof. Since $N$ is a GNF, for each $n \in N$ there is a unique $x \in N$ such that $n 0$ $=n 0 x n 0, x=x n 0 x$. Both 0 and $n 0$ satisfy the above equations. So by uniqueness $0=n 0$. Thus $N$ is zerosymmetric.

By [2, Theorem 1.2, p. 130] $N$ is a GNF if and only if $N$ is regular and idempotents commute. Recall that $N$ is called strongly regular if for each $a \in N$ there exists $b \in N$ such that $a=b a^{2}$. For a brief discussion of these near-rings, see [6], [7] and [8]. In [7], a near-ring $N$ is called subcommutative if $a N=N a$ for all $a \in N$.

Lemma 2. If $N$ is a $G N F$, then $N$ has no non-zero nilpotent elements. 
Proof. Let $a \in N, a^{2}=0$, and let $a$ have inverse $b$. Then $b^{2}=b a b b a b=b b a a b b=0$, since $a b, b a$ are idempotents and hence commute. Also $b a(b a+b)$ is an inverse for $a$, so $b a(b a+b)=b$ by uniqueness. Thus $0=b^{2}=b a(b a+b) b=b a b a b=b a b=b$. So $a$ must be 0 .

We are now ready to prove our main theorems.

Theorem 1. The following are equivalent:

(i) $N$ is a GNF:

(ii) $N$ is regular and each idempotent is central.

(iii) $N$ is regular and subcommutative.

Proof. (i) $\Rightarrow$ (ii). Let $e=e^{2} \in N$ and $a, b \in N$. Since $e^{2}=e,(a-a e) e=0$. By [9, Chapter $9 \mathrm{a}$ and $9 \mathrm{~b}]$, since $N$ has no non-zero nilpotent elements by Lemma $2,(a-a e) b e=0$, so $a b e=a e b e$. But $(e b-e b e) e=0$. For the same reason, $e b(e b-e b e)=0$, $e b e(e b-e b e)=0$ so $(e b-e b e)^{2}=0$ and $e b=e b e$. Thus $a b e=a e b$. Since $N$ is regular, $a=f a$ where $f$ is a suitable idempotent. So $a e=f a e=f e a=e f a=e a$ as idempotents commute. So (ii) holds.

(ii) $\Rightarrow$ (iii). Let $a \in N$. Since $N$ is regular, $a=a x a$ for some $x \in N$. Since $a x$ and $x a$ are idempotents, by (ii) we have $a N=a x a N=a N x a \subseteq N a=N a x a=a x N a \subseteq a N$. Thus $a N$ $=N a$ for all $a \in N$.

(iii) $\Rightarrow$ (i). Let $e, f$ be idempotents. Then $N e=e N$. So there exist $x, y$ in $N$ such that $f e=e x$ and $e f=y e$. Hence $e f e=f e=e f$. So $e f=f e$ and $N$ is a GNF.

Corollary 1. Every $G N F$ is a strongly regular near-ring.

Proof. By (ii) $a=a b a=b a^{2}$ since $b a$ is an idempotent, where $b$ is the inverse of $a$.

In [10], Raphael showed that in a strongly regular ring $R$, for each $0 \neq a \in R$ there exists a unique $b \in R$ such that $a=a b a$ and $b=b a b$. Now the converse follows from Corollary 1 . Thus in the case of rings the notions "strong regularity" and "GNF" are equivalent. In general the converse of Corollary 1 does not hold in near-rings.

Example 1. Let $(N,+)$ be any group. Define multiplications on $N$ as follows:

$$
\begin{gathered}
a b=a \text { for all } a \text { and } 0 \neq b \text { in } N \\
a 0=0 \text { for all } a \text { in } N .
\end{gathered}
$$

Then clearly $N$ is strongly regular but not GNF.

Corollary 2. Every homomorphic image of a GNF is again a GNF.

The definition of a GNF shows that the properties are preserved under homomorphisms.

Combining Theorem 1 and a result of Ligh [5], we have the following: 
Corollary 3. Every GNF is isomorphic to a subdirect product of near-fields and hence $(N,+)$ is abelian.

Theorem 2. $N$ is a GNF and integral if and only if $N$ is a near-field.

Proof. Suppose $N$ is a GNF and integral. Then clearly each non-zero idempotent is a right identity of $N$. If $e, f$ are non-zero idempotents then $f=f e=e f=e$. Thus $N$ has a unique non-zero idempotent, say $e$. Let $0 \neq a \in N$. Then $a=a x a$ for some $x \in N$, ax an idempotent. So $a x=e$ and $e$ is the identity of $N$. Now, by Theorem 3 of [1], $N$ becomes a near-field. The converse is immediate.

Combining Theorem 2 and [9, Corollary 9.38], we get

Corollary 4. Suppose $N$ is subdirectly irreducible. Then $N$ is a GNF if and only if $N$ is a near-field.

In general every GNF is not a near-field.

Example 2. Take a near-field $N$. Then the direct sum of $N$ with itself is a GNF, but not a near-field.

Corollary 5. Suppose for each $0 \neq a$ in $N$ there exists a unique $b \in N$ such that $a=a b a$. Then $N$ is a near-field.

Proof. We first show that $N$ has no zero divisors. Let $a, b \in N$ with $a b=0$ and $b \neq 0$. Then $b=b x b$ for some unique $x \in N$. Now $b(x-a) b=b x b=b$. Hence by the uniqueness of $x$, we have $a=0$. Thus $N$ has no zero divisors. Clearly $N$ is a GNF and hence a nearfield by Theorem 2 .

The following result is an immediate consequence of Corollary 5.

Corollary 6 (Ligh [4]). Let $R$ be a dg near-ring with more than one element. Then $R$ is $a$ division ring if and only if for each $0 \neq a \in R$ there exists $a$ unique $b \in R$ such that $a=a b a$.

In [7], a near-ring $N$ is called left simple if for each $0 \neq a \in N, N a=N$. Clearly a left simple near-ring contains no zero divisors.

Theorem 3. Suppose $N$ has dcc on ideals. Then $N$ is a GNF if and only if $N$ $=N_{1} \oplus \ldots \oplus N_{k}$ where each $N_{i}$ is a near-field.

Proof. Following the proof of [3,Theorem 3.2], we can easily show that the intersection of all maximal ideals is $\{0\}$. Since $N$ has dcc on ideals, there exist maximal ideals $I_{1}, \ldots, I_{n}$ such that $\bigcap_{k=1}^{n} I_{k}=\{0\}$. But from [9, Theorem 2.50, p. 57] $N$ is the direct sum of finitely many simple near-rings. Each summand is a GNF by Corollary 2, hence a near-field by Corollary 4 . The converse is clear. 
Corollary 7. Suppose $N$ is a GNF and satisfies dcc on ideals. Then

(i) $N$ has the identity,

(ii) $a(-b)=(-a) b=-a b$ for all $a, b$ in $N$.

Acknowledgement. I wish to thank my Research Director $\operatorname{Dr} Y$. V. Reddy for suggesting this problem and his valuable guidance. Also I take this opportunity to thank Prof. D. Ramakotaiah for his useful comments on this paper.

\section{REFERENCES}

1. J. C. Beidleman, A note on regular near-rings, J. Indian Math. Soc. 33 (1969), 207-210.

2. J. M. Howle, An Introduction to Semigroup Theory (Academic Press, New York, 1976).

3. M. J. Johnson, Radicals of regular near-rings, Monatsh. Math. 80 (1975), 331-341.

4. S. Ligh, On division near-rings, Canad. J. Math. 21 (1969), 1366-1371.

5. S. LiGH, On regular near-rings, Math. Japon. 15 (1970), 7-13.

6. G. Mason, Strongly regular near-rings, Proc. Edinburgh Math. Soc. 23 (1980), 27-35.

7. C. V. L. N. MuRTY, Structure and ideal theory of strongly regular near-rings, Communicated to Proc. London Math. Soc. (1982).

8. C. V. L. N. MurTy, On strongly regular near-rings II, Communicated to the International Symposium, New Delhi (1982).

9. G. PIlz, Near-rings (North-Holland, Amsterdam, 1977).

10. R. Raphael, Some remarks on regular and strongly regular rings, Canad. Math. Bull. 17 (1975), 709-712.

Department of Mathematics

NagaruUna UnIVERSITY

Nagaruunanagar-522 510

A.P. INDIA 